Original Contribution

\title{
EFFECT OF HYPERTONICITY ON THE DYNAMIC STATE OF ERYTHROCYTE SPECTRIN NETWORK AS REVEALED BY THERMAL DIELECTROSCOPY
}

\author{
B. Paarvanova, I. Ivanov* \\ Department of Physics, Biophysics, Roentgenology and Radiology, Medical Faculty, \\ Trakia University, Stara Zagora, Bulgaria
}

\begin{abstract}
Hypertonicity-induced alteration of the spectrin network under the erythrocyte membrane (EM) has been proposed as the cause of hypertonic cryohemolysis, however, the exact mechanism is not known. With this in mind we applied thermal dielectroscopy to evaluate the dynamic state of spectrin network of human erythrocytes and isolated EMs as affected by the osmotic pressure of suspension media (10 mM $\mathrm{NaCl}$ and varying concentrations of mannit). The method, applied on a heated suspension, detected abrupt changes in the complex impedance, $\Delta Z^{*}=\Delta Z_{\mathrm{re}}+\mathrm{j} \Delta Z_{\mathrm{im}}$, at the spectrin denaturation temperature, $\mathrm{T}_{\mathrm{A}}(49.5$ $\left.{ }^{\circ} \mathrm{C}\right)$. The Nyquist plot $\left(-\Delta Z_{\text {im }}\right.$ vs $\Delta Z_{\text {re }}$ ) of these changes indicated two arcs, i.e., two relaxations of the mobile dipoles on intact spectrin network, one centered at $2.5 \mathrm{MHz}$ and the other at $0.3 \mathrm{MHz}$. Increasing the tonicity from 300 to 500 mOsm both arcs were inhibited. Similar inhibition of the Nyquist plot arcs was obtained by the selective dissociation of spectrin-ankyrin-band 3 bridge of EM. Above $500 \mathrm{mOsm}$ the arcs merged indicating a single relaxation. Both effects of hypertonicity were reversible upon reverting to isotonicity. It is concluded that hypertonicity could lead to a reversible detachment of spectrin skeleton from the integral proteins and inhibition of the segmental motion of spectrin network.
\end{abstract}

Key words: erythrocyte membrane, spectrin skeleton, dipole polarization, hypertonic cryohemolysis.

\section{INTRODUCTION}

The osmotic pressure of hypertonic media elicits changes in the structure and function of cellular membranes. In mammals, the interstitium of the renal medulla is in hypertonic environment whose hypertonicity imposes osmotic gradient for water reabsorption and renal concentrating mechanism. At the same time, hypertonicity induces deleterious effects and apoptosis on local cells (1) and provides a local signal for tissue-specific gene expression and differentiation of the renal medulla (2).

Essential to the unique mechanical properties of human erythrocytes is the erythrocyte membrane (EM) consisting of a lipid bilayer with intercalated integral proteins and supported from inside by a under-membrane cytoskeleton of peripheral proteins, among them spectrin and ankyrin (Figure 1). Spectrin is a filamentous long (about $200 \mathrm{~nm}$ )

\footnotetext{
*Correspondence to: Ivan Tanev: Dept. Physics, Biophysics, Rentgenology and Radiology, Medical Faculty, Thracian University, Armeyska Str. 11, Stara Zagora 6000, Bulgaria. E-mail: ivanov_it@gbg.bg
}

multidomain protein that comprises about $30 \%$ $(\mathrm{w} / \mathrm{w})$ of membrane protein content. EM contains another two major integral proteins, band 3 and glycophorin $\mathrm{C}$, both attached to the spectrin-based under-membrane cytoskeleton. The first attachment site includes spectrin glycophorin $\mathrm{C}$ bridge. The second, predominant attachment site is the spectrin ankyrin - band 3 bridge, responsible for the deformability and stability of erythrocytes (3). In a single cell there are about 100 thousand spectrin - ankyrin - band 3 bridge, most of which can be selectively severed binding DIDS to the outside aspect of band 3 (4) or suspending erythrocytes in alkaline $\mathrm{pH}$ (5).

The plasma membrane of human erythrocytes also alters on contact with hyperosmotic media. This alteration prevents normal adaptation of EMs to consequent cooling (5 ${ }^{\circ} \mathrm{C}$ ) and results in hemolysis termed hypertonic cryohemolysis, or cold shock and heat shock hemolysis (7). The molecular mechanism of the hypertonicity-induced alteration of EM and the related cryohemolysis has not been elucidated so far. Data have been presented indicating that the under-membrane spectrin cytoskeleton of EM and its interaction with the 
lipid bilayer is involved in hypertonic cryohemolysis (8). Nevertheless, hypertonic cryohemolysis is exploited as a model experiment in evaluating the damaging factors involved in the long-term, low-temperature storage of cells and tissues, in the hemolysis due to inappropriate storage conditions or inappropriate processing of blood for transfusion (9), as well as a useful technique for identifying hereditary spherocytosis (1011).

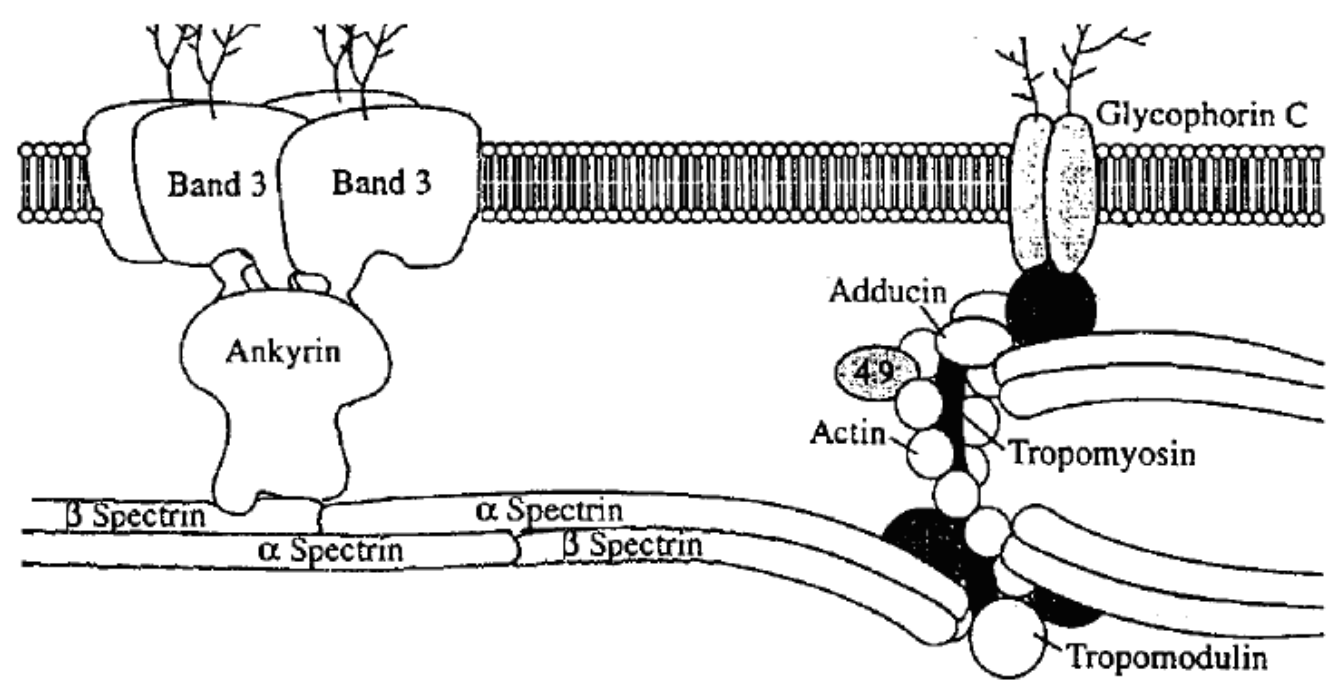

Figure 1. Diagram of the spectrin - band 3 bridge and of the spectrin-glycophorin bridge on the plasma membrane of human erythrocytes (6).

With above in mind we used the method of thermal dielectroscopy to study the heat denaturation of under-membrane spectrin network of human erythrocytes, suspended in media with constant composition and different tonicities. The denaturation of spectrin filaments at $49.5^{\circ} \mathrm{C}\left(\mathrm{T}_{\mathrm{A}}\right)$ leads to dramatic loss in elasticity and deformability of erythrocyte membrane which is indicative of complete aggregation and immobilization of denatured spectrin and loss in its dielectric polarizability. The method detects abrupt changes in the complex impedance of erythrocyte suspension at $\mathrm{T}_{\mathrm{A}}$ (12). These changes reflect the polarizability of intact spectrin skeleton as this polarizability vanishes after the heat denaturation of spectrin. Because this polarizability is due to the mobile dipoles on spectrin skeleton, it is frequency dependent reflecting the dipole relaxation and the dynamic state of intact spectrin skeleton. To describe the relaxation of spectrin dipoles and, hence, the dynamic state of intact spectrin skeleton we obtained the Nyquist plot of impedance changes at $T_{A}$ for erythrocytes and isolated erythrocyte membranes, suspended at various tonicities. The presented results demonstrate that hypertonic media inhibits the segmental motions of spectrin filaments and could dissociate the spectrin cytoskeleton from the lipid bilayer.

\section{MATERIALS AND METHODS Materials}

4,4'-diiso-thiocyanato stilbene-2,2'-disulfonic acid (DIDS), $\mathrm{MgCl}_{2}, \mathrm{NaCl}$, mannitol and phosphate buffer were purchased from Sigma Chemicals Co, St. Louis, MO, USA.

\section{Isolation of erythrocytes and severance of spectrin-ankyrin-band 3 bridge}

Intact erythrocytes were isolated by centrifugation $(500 \mathrm{x} \mathrm{g}, 5 \mathrm{~min})$ from freshly drawn heparinized blood of healthy donors. The upper layer of white blood cells was discarded. Prior to usage the isolated cells were thrice washed in 10 volumes of washing medum, containing $10 \mathrm{mM} \mathrm{NaCl}$ and the concentration of mannit corresponding to the indicated osmoticity.

Erythrocytes with disconnected linkage between spectrin, ankyrin and band 3 were prepared in two ways. The first way consists in treating intact erythrocytes with DIDS. DIDS is a membrane-impermeable, highly specific covalent inhibitor of band 3 protein (the anion exchanger, AE1), the major integral protein of erythrocyte membrane responcible for the anion transport across EM (13). Washed erythroctes were incubated in a solution containing $0.15 \mathrm{M} \mathrm{NaCl}, 50 \mu \mathrm{M}$ DIDS and 5 $\mathrm{mM}$ sodium phosphate buffer, $\mathrm{pH} 8.2$, at $10 \%$ hematocrit, $23{ }^{\circ} \mathrm{C}$ for $10 \mathrm{~min}$. Treated cells 
were washed two times with 10 volumes of isotonic washing medium to remove the unreacted DIDS. Under these conditions, more than $95 \%$ of the DIDS resides on band 3 (14) resulting in severance of the major bilayer skeleton bridge between band 3, ankyrin and spectrin (4).

The second way to dissociate the linkage between band 3 and spectrin consists in suspending intact erythrocytes in isotonic medium of mannitol and $5 \mathrm{mM} \mathrm{NaCl}$, containing $10 \mathrm{mM}$ phosphate or Tris buffer, hematocrit 0.20 . At $\mathrm{pH} 9.3$ the cytoplasmic domain of band 3 sustains conformation change causing detachment of band 3 from ankyrin, and thereby from spectrin cytoskeleton (5).

\section{Preparation of isolated and resealed erythrocyte ghost membranes (EMs)}

Erythrocyte ghost membranes (EMs), resealed with $75 \mathrm{mM} \mathrm{NaCl}$ and $150 \mathrm{mM}$ mannit, were prepared as described previously (15). Briefly, cold $\left(1{ }^{\circ} \mathrm{C}\right)$ suspension of washed erythrocytes (intact or DIDS-treated), hematocrit 0.80 , was vigorously diluted in 15 volumes of $1{ }^{\circ} \mathrm{C}$-cold hypotonic solution, containing $2 \mathrm{mM} \mathrm{MgCl}_{2}$ and $5 \mathrm{mM}$ phosphate buffer, $\mathrm{pH} 7.8$ and left at cold for $5 \mathrm{~min}$. A proper volume of cold 0.75 $\mathrm{M} \mathrm{NaCl}$ and of cold 1.2 M mannit solutions were added to the hemolysate in order to obtain final concentrations of $75 \mathrm{mM} \mathrm{NaCl}$ and $150 \mathrm{mM}$ mannit. The EMs were resealed (37 $\left.{ }^{\circ} \mathrm{C}, 20 \mathrm{~min}\right)$, isolated $(3000 \mathrm{x} \mathrm{g}, 10 \mathrm{~min})$ and once washed in excess $(1: 20)$ volume of the resealing medium.

\section{Impedance spectroscopy of heated erythrocyte suspensions}

To conduct the thermal dielectroscopy study $50 \mu \mathrm{l}$ of erythrocytes or EMs were suspended in $50 \mu \mathrm{l}$ medium, containing $10 \mathrm{mM} \mathrm{NaCl}$ and mannit with the indicated concentration. The suspension was put in a sample cuvette and heated from 20 to $60^{\circ} \mathrm{C}$ at $2.0^{\circ} \mathrm{C} / \mathrm{min}$ heating rate as previously described (15). During heating the complex impedance, $Z^{*}=Z_{\mathrm{re}}+\mathrm{j}$. $Z_{\text {im }}$, of the suspension was measured and separated into its real $\left(\mathrm{Z}_{\mathrm{re}}\right)$ and imaginary $\left(\mathrm{Z}_{\mathrm{im}}\right)$ parts. Here, $\mathrm{j}$ is the imaginary unit, $\mathrm{j}=(-1)^{0.5}$. $Z_{\mathrm{re}}$ and $\mathrm{Z}_{\mathrm{im}}$ both abruptly changed at the spectrin denaturation temperature $\left(\mathrm{T}_{\mathrm{A}}=49.5\right.$ ${ }^{\circ} \mathrm{C}$ ) and these changes, measured at various frequencies, represented the basic data of this study. The core instrument was a Solartron 1260 Impedance Analyzer (Schlumberger Instruments, Hampshire, England) interfaced to Toshiba PC using the Miniscan software. $Z^{*}$ was measured at the indicated frequencies between 0.05 and $13 \mathrm{MHz}$, scanned serially with integration time of $0.5 \mathrm{~s}$ and duration of each scan about $10 \mathrm{~s}$.

In order to decrease electrode polarization we used platinum electrodes, low suspension conductance (about $40 \mu \mathrm{S}$ ), high hematocrit value $(0.60)$, low electrode voltage $(80 \mathrm{mV})$ and frequencies above $50 \mathrm{kHz}$.

\section{RESULTS AND DISCUSSION}

When the studied suspension of intact erythrocytes was heated, the imaginary part, $Z_{i m}$, of its complex impedance, $Z^{*}$, exhibited sigmoid change at the spectrin denaturation temperature $\mathrm{T}_{\mathrm{A}}$ (Figure 2). The magnitude and direction of this change depended on the measuring frequency. Similar frequencydependent changes in the real part, $Z_{r e}$, of $Z^{*}$ were also registered at $\mathrm{T}_{\mathrm{A}}$ (not shown). Exactly the same sigmoid changes were registered in the impedance of suspensions, containing resealed EMs (not shown). The single midpoint temperature, $T_{A}$, of these changes slightly depended on the heating rate and at heating rates extrapolated to zero its value tended to $49.5 \pm 0.2{ }^{\circ} \mathrm{C}$, the known temperature of spectrin denaturation by heat (16). Using erythrocytes with specifically pre-modified spectrin the threshold changes in $Z_{r e}$ and $Z_{i m}$ at $T_{A}$ were directly ascribed to the thermal denaturation of spectrin and related change in the dipole polarization of spectrin network (12).

The value of the detected change in $Z_{i m}$ at $T_{A}$ was initially defined as $\Delta Z_{i m}=\left(Z_{i m}\right)_{\text {native }}-$ $\left(Z_{\text {im }}\right)_{\text {denatured }}$, where $\left(Z_{\text {im }}\right)_{\text {native }}$ and $\left(Z_{\text {im }}\right)_{\text {denatured }}$ are the imaginary part of suspension impedance at the native state of spectrin (at a temperature 3 ${ }^{\circ} \mathrm{C}$ less than $T_{A}$ ) and at the denatured state of spectrin (at a temperature $3^{\circ} \mathrm{C}$ greater than $T_{A}$ ), respectively. This change, however, levels off within a substantial temperature interval of about $6{ }^{\circ} \mathrm{C}$ where it is superimposed on the continuous, thermally induced variation of electrolyte conductivity. To compensate for the temperature effect on conductivity, which had no relation to the temperature-induced denaturation at $T_{A}$, the change in $Z_{i m}$, taking place over an equal temperature interval prior the denaturation at $T_{\mathrm{A}}$, was similarly calculated and subtracted from the initial $\Delta Z_{i m}$. The change in $Z_{r e}$ at $T_{\mathrm{A}}$, i.e., $\Delta Z_{r e}$, was likewise determined and corrected. Depending on the measuring frequency, the values of $\Delta Z_{r e}$ and $\Delta Z_{i m}$ had different magnitudes and signs. 
PAARVANOVA B., et al.

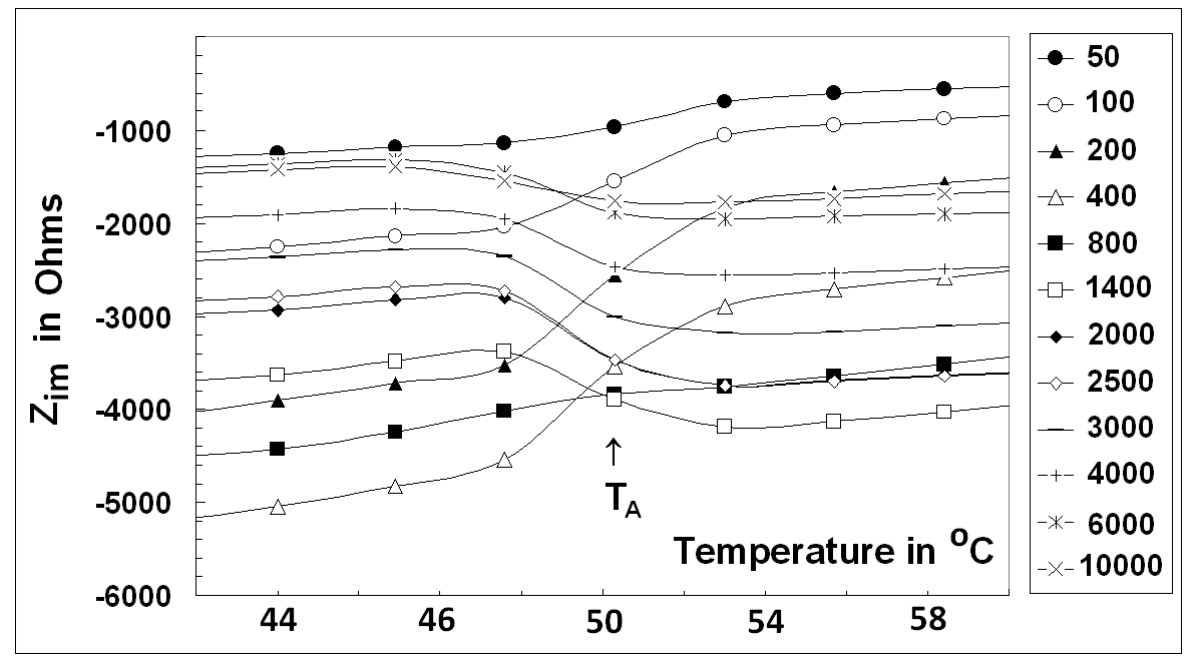

Figure 2. Temperature profile of the imaginary component of suspension impedance, $Z_{\text {im. }}$ The suspensions contained either erythrocytes or resealed EMs. Shown is the change in $Z_{\text {im }}$, ie $\Delta Z_{\text {im }}=\left(Z_{\text {im }}\right)_{\text {native }}-\left(Z_{\text {im }}\right)_{\text {denatured }}$, at the spectrin denaturation temperature, $\mathrm{T}_{\mathrm{A}}$, which is indicated by arrow. The suspension medium was isotonic solution of $10 \mathrm{mM} \mathrm{NaCl}$ and mannit. The hematocrit and heating rate were 0.50 , and $2.0^{\circ} \mathrm{C} / \mathrm{min}$, respectively. The measuring frequencies are shown in the insert rightward.

In the Introduction section it was explained that the values of $\Delta Z_{i m}$ and $\Delta Z_{r e}$ reflect the mobility of dipoles on the intact spectrin network and, hence, the segmental mobility of intact spectrin filaments. This segmental mobility could be investigated studying the frequency dependence of $\Delta Z_{i m}$ and $\Delta Z_{r e}$. The complex plane (Nyquist) plot of $-\Delta Z_{i m}$ vs $\Delta Z_{r e}$ (Figure 3) expressed this frequency dependence in the form of two semicircle acrs, one positive above the argument axis, and a second one negative below the argument axis. Exactly the same plot was obtained heating suspensions of resealed EMs (Figure 3).

Theoretically each semicircle arc in Figure 3 corresponds to the frequency relaxation of a polarization mechanism of Debye type, i.e. having a single relaxation time (17). The negative semicircle arc exhibited the $-\Delta Z_{i m}$ vs $\Delta Z_{r e}$ interrelationship at frequencies $(1-10$ $\mathrm{MHz}$ ) which allowed the incident electric field to fully penetrate into the cytosole of erythrocytes and resealed EMs. Thus, this semicircle arc corresponded to a relaxation mechanism associated to the cytosolic side of erythrocyte plasma membrane. According to previous study (12) this relaxation could involve dipoles associated to the highly flexible segments of spectrin filaments. The critical frequency, $f_{\text {delta, of }}$ this relaxation, designated by an arrow in Figure 3, was determined about $2.5 \mathrm{MHz}$.

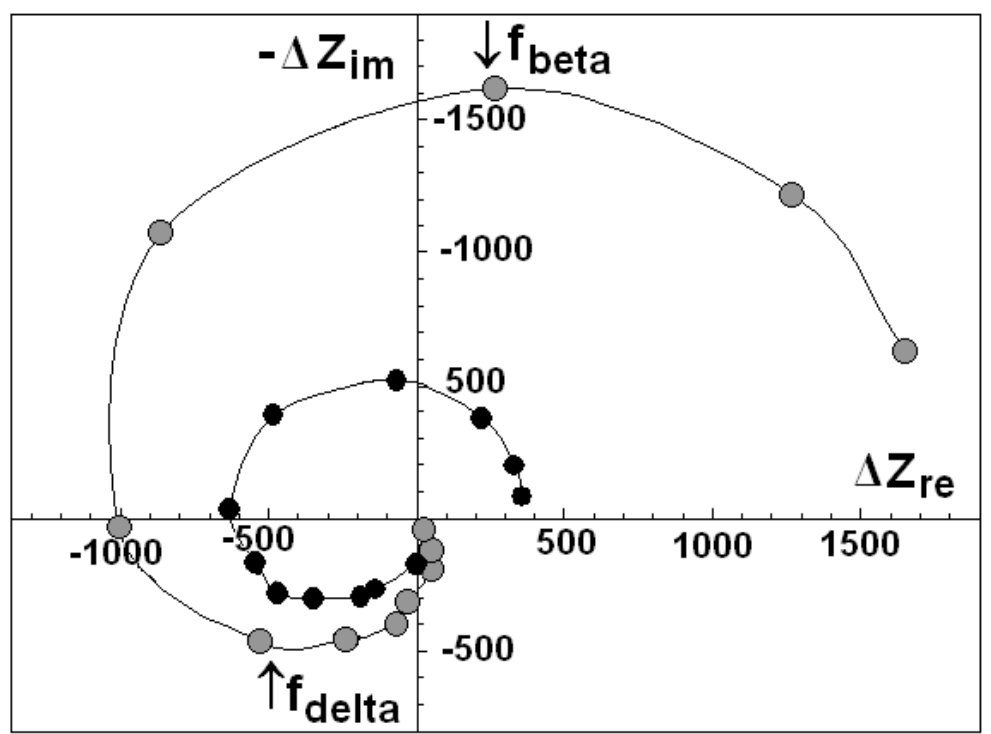

Figure 3. Complex impedance (Nyquist) plot at the temperature of spectrin denaturation, $\mathrm{T}_{\mathrm{A}}$. The suspension contained intact erythrocytes or resealed EMs (gray circles) and DIDS-treated erythrocytes or EMs isolated from DIDS-treated erythrocytes (black circles). The $\Delta Z_{\mathrm{im}}$ and $\Delta \mathrm{Z}_{\mathrm{re}}$ are the changes in the imaginary and real components of suspension impedance, $Z^{*}$, associated with the denaturation of spectrin. All other details are as indicated for Figure 2. 
The positive semicircle arc in Figure 3 exhibited the $-\Delta Z_{i m}$ vs $\Delta Z_{r e}$ interrelationship at low frequencies $(0.05-1.0 \mathrm{MHz})$. The critical frequency, $\mathrm{f}_{\text {beta }}$, of the positive semicircle arc, designated by an arrow in Figure 3, was determined about $0.30 \mathrm{MHz}$, i.e., 8 times lower compared to $f_{\text {delta. }}$. In a separate experiment, the critical frequency of the accompanying interfacial polarization was determined to have the same value of $0.30 \mathrm{MHz}$ at $46{ }^{\circ} \mathrm{C}$ (not shown). The interfacial polarization is due to alternating accumulation of charges, in phase
PAARVANOVA B., et al. with the incident field, on both sides of plasma membrane. The charge accumulation at membranes is slow process and at a sufficiently high, so called critical, frequency of the field it fades away; this is the so called beta relaxation. Increasing the $\mathrm{NaCl}$ concentration of suspension medium from 10 $\mathrm{mM}$ to $75 \mathrm{mM}$, the critical frequency of beta relaxation and the critical frequency of the positive semicircle arc both increased from $0.30 \mathrm{MHz}$ to $1.0 \mathrm{MHz}$, remaining always equal to each other (not shown).

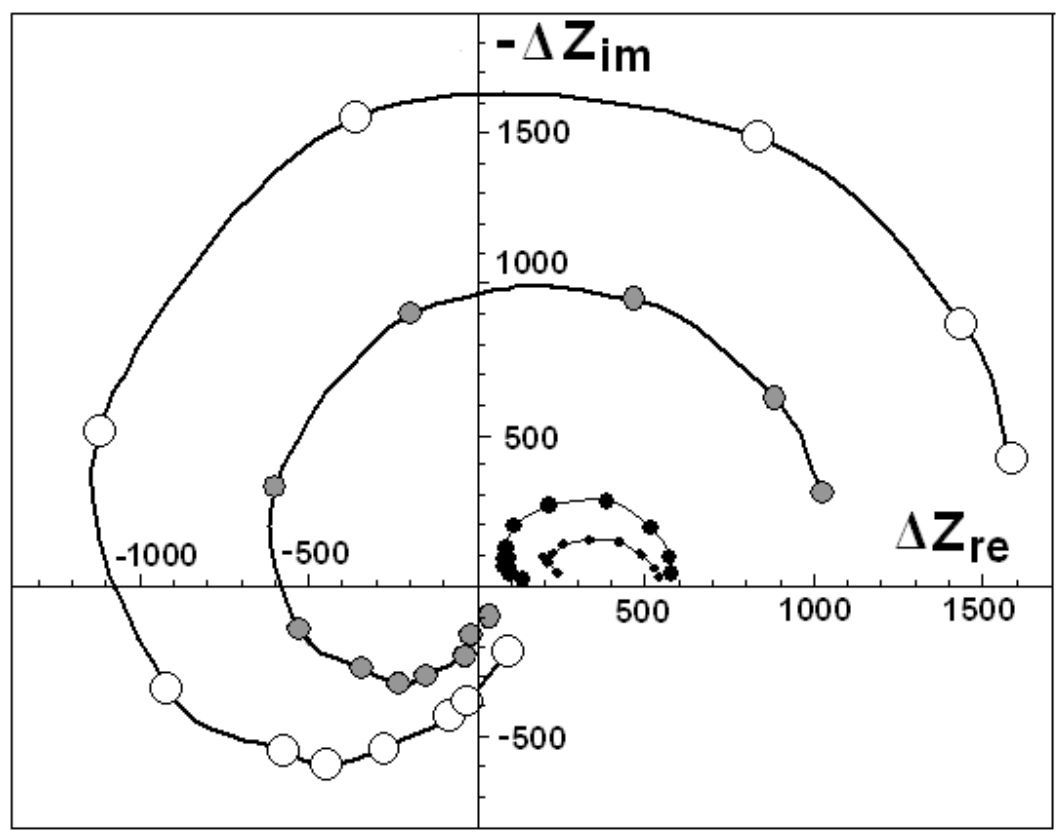

Figure 4. Effect of hypertonicity on the complex impedance (Nyquist) plot of intact erythrocytes at the temperature of spectrin denaturation. The erythrocytes were suspended in media containing $10 \mathrm{mM} \mathrm{NaCl}$ and different concentrations of mannit producing osmotic pressure in mOsmols: 280 (open circles), 430 (gray circles), 580 (black circles) and 1180 (small black circles). All other details are as indicated for Figure 2.

Based on above results, the positive semicircle arc was assumed to reflect the association of the under-membrane spectrin skeleton to the inner aspect of the lipid bilayer. This assumption is supported by the results, obtained with erythrocytes with severed spectrin - band 3 bridge. The severance of this bridge was obtained through DIDS treatment of erythrocytes (4) and by suspending the erythrocytes in isotonic media with pH 9.3 (5). DIDS treatment of erythrocytes constitutes a noninvasive method by which the spectrin band 3 bridge can be severed and the resulting effect on the complex impedance plot of erythrocyte suspension examined. This bridge involves two types of linking sites between band 3 and ankyrin, one with high and another with low affinity. DIDS binding to band 3 has been shown to induce loss of all low affinity and roughly half of all high affinity linking sites between band 3 and ankyrin (4). The complex impedance plot of DIDS-treated erythrocytes and of EMs isolated from DIDStreated erythrocytes both showed that DIDS binding was accompanied by strong reduction of the positive semicircle arc (Figure 3). Similar outcome was obtained with intact erythrocytes and resealed EMs, suspended in isotonic alkaline medium of mannit and 10 $\mathrm{mM}$ phosphate or Tris buffer (not shown). Reduction of the positive semicircle arc was progressively enlarged with increasing the $\mathrm{pH}$ of outside medium from $\mathrm{pH} 8.0$ to $\mathrm{pH} 9.3$ and at $\mathrm{pH} 9.3$ the effect was equal to that of DIDS (Figure 3). Both lines of evidence indicate that the positive arc of the complex impedance plot at spectrin denaturation temperature sensed the status of spectrin - band 3 linkage of the erythrocyte plasma membrane. 


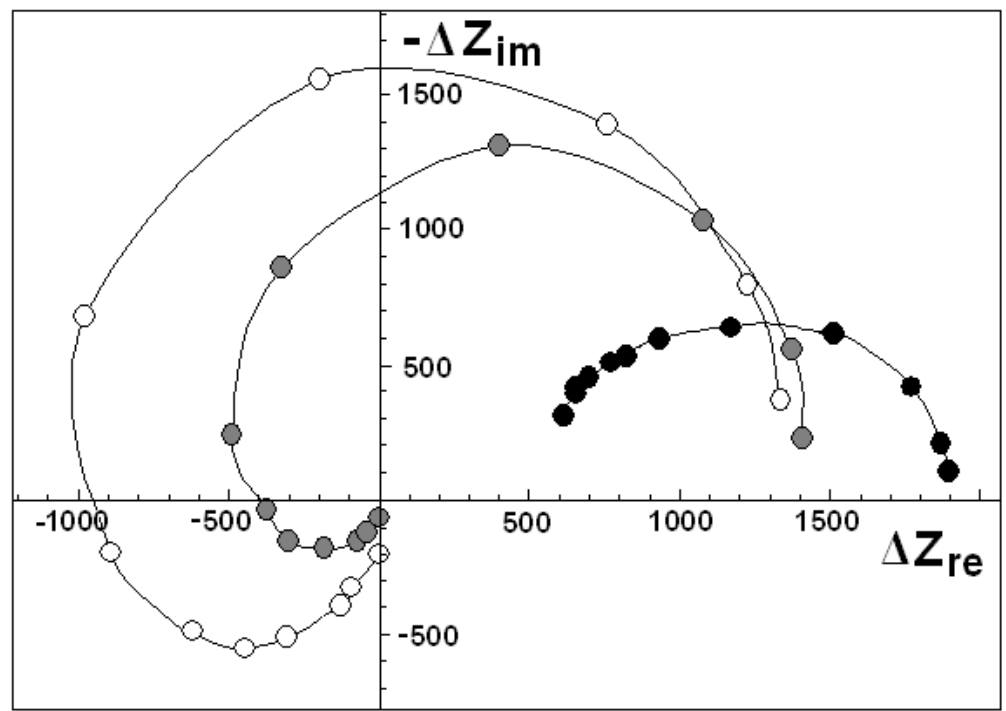

Figure 5. Reversibility of the effect of hypertonicity on the complex impedance (Nyquist) plot of intact erythrocytes at the temperature of spectrin denaturation. The erythrocytes were suspended in media containing $10 \mathrm{mM} \mathrm{NaCl}$ and different concentrations of mannit producing osmotic pressure in mOsmols: 280 (open circles), 880 (black circles). The gray circles indicate erythrocytes, initially suspended in $10 \mathrm{mM} \mathrm{NaCl}$ and mannit producing $880 \mathrm{mOsm}$ for $30 \mathrm{~min}$ and reverted to a medium of $10 \mathrm{mM} \mathrm{NaCl}$ and mannit producing $280 \mathrm{mOsm}$. All other details are as indicated for Figure 2.

The above conclusion was exploited in the studying the effect of hypertonicity on the plasma membrane of erythrocytes. For this purpose the erythrocytes were washed and suspended in various hyperosmotic media containing the same concentration of $10 \mathrm{mM}$ $\mathrm{NaCl}$ and respective concentration of mannit to obtain the indicated hypertonicity. Since mannit is impermeable to the erythrocyte membrane, the cells osmotically shrunk and their membranes altered under the impact of hypertonicity. Figure 4 shows that the effect of hypertonicity on the $-\Delta Z_{i m}$ vs $\Delta Z_{r e}$ (i.e., Nyquist) plot of intact erythrocytes had two separate stages. The first stage was demonstrated at tonicities between 300 and 500 mOsm while the second stage was apparent above $500 \mathrm{mOsm}$. With increasing the hypertonicity from 300 to about $500 \mathrm{mOsm}$ the two relaxation arcs were progressively suppressed however, the second arc remained below the ordinate axis. Similar suppression was obtained with isolated EMs, resealed with isotonic medium of $75 \mathrm{mM} \mathrm{NaCl}$ and mannit (not shown). Thus, the effect of moderate hypertonicity $(300-500 \mathrm{mOsm})$ on intact erythrocytes and resealed EMs was similar to the effects of DIDS and alkaline media, and therefore, could be possibly explained by the disassociation of spectrin - band 3 bridges.

At tonicities above $500 \mathrm{mOsm}$, both arcs merged into a singe arc that was placed above the ordinate axis (Figure 4 and Figure 5). This possibly indicated merger of the two relaxations due to a tonicity-induced transition in the spectrin network. This additional effect was absent in
DIDS-treated erythrocytes and in isolated EMs (not shown) and, consequently, it could not be due to the hemoglobin present in the cytosole of erythrocytes.

Interestingly enough, the above indicated effects of hypertonicity (300 - $1200 \mathrm{mOsm}, 30 \mathrm{~min}$ ) on the Nyquist plot of intact erythrocytes was almost entirely reversible. Reverting the tonicity from $900 \mathrm{mOsm}$ to $300 \mathrm{mOsm}$ the suppression of relaxation circles and the shift of Nyquist plot were removed and the Nyquist plot of erythrocytes restored its shape (Figure 5). This reversibility is in line with the assumed disassociation of spectrin - band 3 bridge at hypertonic conditions and could be explained as follows. Human erythrocyte band 3 becomes rapidly and reversibly phosphorylated following exposure of erythrocytes to hypertonic media (18). The driving force for this phosphorylation reaction is the decrease in cell volume that activates specific, membrane-bound, tyrosine kinase. The phosphorylated site is identified as Tyr- 8 , located at the extreme $\mathrm{N}$-terminus of the cytoplasmic domain of band 3 , responcible for the binding of band 3 to ankyrin. Consequently, the phosphorylation of the Tyr- 8 residue of band 3 immediately disassociates the spectrin - band 3 bridge (19). As the phosphorylation reaction is reversible on restoring the initial volume of erythrocytes (18) the spectrin - band 3 bridge should reassociate in accordance with the result shown in Figure 5.

The assumed in this study reversible disassociation of spectrin - band 3 bridge at hypertonic conditions is supported by the data of other authors that chemical pre-modifications of EM, which dissociate band 3 from undermembrane cytoskeleton, increase hypertonic 
cryohemolysis (20) and detergent - induced hemolysis (21).

\section{CONCLUSSION}

The changes in the dielectric properties of erythrocyte plasma membrane, concomitant to the thermal denaturation of spectrin at $49.5{ }^{\circ} \mathrm{C}$, were used to investigate the relaxation of mobile dipoles on intact spectrin network. The mobility of these dipoles, reflecting the segmental motions of spectrin filaments, was reversibly subdued upon increase and decrease of the osmolarity of cell suspension. This outcome could involve the detachment of spectrin network from the band 3 protein. The results are relevant to elucidating the mechanism of hypertonic cryohemolysis and the effects of hyperosmoticity on cells.

\section{ABBREVIATIONS}

DIDS, $\quad 4,4^{\prime}$-di-isothiocyanostilbene-2,2' disulphonate; EM, erythrocyte ghost membrane resealed with $75 \mathrm{mM} \mathrm{NaCl}$ and $150 \mathrm{mM}$ mannit.

\section{REFERENCES}

1. Horio, M., Ito, A., Matsuoka, Y., Moriyama, T., Orita, Y., Takenaka, M. and Imai, E., Apoptosis induced by hypertonicity in Madin Darley canine kidney cells: protective effect of betaine. Nephrol Dial Transplant, Mar; 16(3):483-90, 2001.

2. Kwon, M., S., Lim, S., W. and Kwon, H., M., Hypertonic stress in the kidney: a necessary evil. Physiology (Bethesda),24:186-191, 2009

3. Willardson, B., M., Thevenin, B.,J., Harrison, M., L., Kuster, W., M., Benson, M., D. and Low, P., S., Localization of the ankyrinbinding site on erythrocyte membrane protein, band 3. J Biol Chem., 264(27):15893-15899, 1989.

4. Van Dort, H., M., Moriyama, R. and Low, P., S., Effect of band 3 subunit equilibrium on the kinetics and affinity of ankyrin binding to erythrocyte membrane vesicles. $J$ Biol Chem, 273(24):14819-14826, 1998.

5. Low, P., Willardson, B., Mohandas, N., Rossi, M. and Shohet, S., Contribution of the band 3-ankyrin interaction to erythrocyte membrane mechanical stability. Blood, 77:1581-1586, 1991.

6. Tse, W., T. and Lux. S., Red blood cell membrane disorders. $\mathrm{Br} \quad \mathrm{J}$ Haematol, 104(1):2-13, 1999.

7. Green, F., A. and Jung, C., Y., Cold-induced hemolysis in a hypertonic milieu. J Membr Biol., 33(3-4):249-262, 1977.

8. Green, F., A., Jung, C., Y., Cuppoletti, J. and Owens, N., Hypertonic cryohemolysis and the cytoskeletal system. Biochim Biophys Acta, 648(2):225-230, 1981.

9. Sowemimo-Coker, S., O., Red blood cells during processing. Transfus Med Rev., 16(1):46-60, 2002.

10.Streichman, S. and Gescheidt, Y., Cryohemolysis for the detection of hereditary
PAARVANOVA B., et al. spherocytosis: correlation studies with osmotic fragility and autohemolysis. Am $J$ Hematol. 58(3):206-212, 1998.

11. King, M., J. and Zanella, A., Hereditary red cell membrane disorders and laboratory diagnostic testing. Int $J$ Lab Hematol, 35(3):237-243, 2013.

12.Ivanov, I., T., Paarvanova, B. and Slavov, T., Dipole relaxation in erythrocyte membrane: Involvement of spectrin skeleton. Bioelectrochemistry, 88:148-155, 2012.

13. Cabantchik, Z., I. and Greger, R., Chemical probes for anion transporters of mammalian cell membranes. Am J Physiol, 262:C803C827, 1992.

14. Jennings, M., L. and Passow, H., Anion transport across the erythrocyte membrane, in situ proteolysis of band 3 protein, and crosslinking of proteolytic fragments by 4,4'diisothiocyano dihydrostilbene-2,2'disulfonate. Biochim Biophys Acta, 554(2):498-519, 1979.

15.Ivanov, I., T., Impedance spectroscopy of human erythrocyte membrane: Effect of frequency at the spectrin denaturation transition temperature. Bioelectrochemistry, 78:181-185, 2010.

16. Brandts,J.,F.,Erickson,L.,Lysko, K., Schwartz, A., T. and Taverna, R., D., Calorimetric studies of the structural transitions of the human erythrocyte membrane. The involvement of spectrin in the A transition.Biochemistry,16:3450-3454, 1977.

17. Kell, D., B., The principles and potential of electrical admittance spectroscopy: An introduction. In: Turner APF, Karube I, Wilson G (eds), Biosensors: fundamenlals and applications, Oxford University Press, pp 427-468, 1987.

18. Minetti, G., Seppi, C., Ciana, A., Balduini, C., Low, P., S., and Brovelli. A., Characterization of the hypertonically induced tyrosine phosphorylation of erythrocyte band 3 . Biochem J, 335:305-311, 1998.

19. Ferru, E., Giger, K., Pantaleo, A., Campanella, E., Grey, J., Ritchie, K., Vono, R., Turrini, F., and Low P., S., Regulation of membrane-cytoskeletal interactions by tyrosine phosphorylation of erythrocyte band 3. Blood, 117(22):5998-6006, 2011.

20. Cherkashina, Ya., O., Effect of Cryoprotectants on Hypertonic Cryohemolysis Rate of Human Erythrocytes with Modified Cytoskeleton-Membrane Complex. Problems of cryobiology, 22 (2):126-132, 2012.

21.Markova, K., V., Ramazanov, V., V. and Bondarenko, V., A., Osmotic Effect On Human Erythrocyte Detergent Lysis. Problems of cryobiology, 19 (3):243-253, 2009. 
PAARVANOVA B., et al. 